\title{
EI gasto social y el voto: análisis de los principales factores que influyen en el comportamiento del gobierno y los votantes Ecuador 2007-2017
}

\section{Social Spending and Voting: Analysis of the Main Factors that Influence the Behavior of the Government and the Voters Ecuador 2007-2017}

\section{Sandra Borja}

Recepción: 07-05-2020

\section{Resumen}

Aceptación: 20-07-2020

Este trabajo analiza el comportamiento de dos actores clave del mercado político, el gobierno y el votante. En específico, se prueban las hipótesis planteadas por teóricos de la Escuela de la Elección Pública, las cuales sostienen que el gobierno y el votante son agentes racionales que al igual que en el mercado intercambian recursos con el objetivo de maximizar su utilidad. De manera general, se evidencia que el gobierno puede usar sus recursos de manera táctica para mantenerse en el poder, además, los sectores más vulnerables mantienen su apoyo por el candidato de Gobierno. Para Ecuador, a través de un modelo econométrico de datos de panel, se prueban estas hipótesis, y se evidencia que el gasto social, al focalizarse en los sectores más vulnerables, fue una variable clave para la estabilidad del gobierno en el período 2007-2017.

Palabras claves: Gobierno, votante, votos, gasto social, racionales.

\section{Abstract}

This work analyzes the behavior of two key actors in the political market, the government and the voter. Specifically, the hypotheses put forward by theorists of the School of Public Choice are tested. These theorists maintain that the government and the voter are rational agents who, like in the market, exchange resources with the aim of maximizing their utility. In general, it is evident that the Government can use its resources tactically to stay in power, and the most vulnerable sectors maintain their support for the Government candidate. For Ecuador, using an econometric panel data model, these hypotheses are tested, and it is evident that social spending, by targeting the most vulnerable sectors, was a key variable for government stability in the period 2006-2017.

Key Words: Government, voter, votes, social spending, rational. 


\section{Introducción}

inicios del siglo, Latinoamérica evidenció una tendencia marcada
hacia los gobiernos de izquierda, quienes principalmente basaron su
política económica y social en el denominado socialismo del siglo XXI. ${ }^{1}$ Este modelo promueve una alta participación del Estado en la actividad económica y posturas asistencialistas, por lo que utiliza el gasto social como una variable primordial para promover el desarrollo económico y social (Dieterich, 2003).

Entre los países que implementaron esta ideología se encuentra el gobierno de Rafael Correa en Ecuador, cuyo mandato duró diez años, convirtiéndose en el más largo de la historia nacional. Por esta razón, este trabajo tiene como fin principal entender los mecanismos por los que se consiguió tal estabilidad y de manera específica si para conseguirla se influyó en la repartición de recursos públicos de manera táctica.

Para lograr este cometido, se pretende analizar teórica y empíricamente el comportamiento del gobierno y del votante durante los años 2007-2017. Para esto, se recurre al análisis teórico de los trabajos realizados por diversos autores que forman parte de la escuela de la elección pública, la cual sostiene que las motivaciones en el proceso político son las mismas que existen en el mercado. De esta manera, se espera que los votantes y gobernantes actúen buscando su propio interés (Buchanan, 2005).

Para entender el comportamiento del gobierno, planteamos que este distribuye sus recursos de manera táctica, específicamente en sectores que resulten ser políticamente rentables. Además, partimos de reconocer al gobierno como un actor riesgo adverso, lo que quiere decir que invierte en aquellos sectores que ya lo apoyaron en las elecciones pasadas. Esta idea se desarrollará más adelante.

Con respecto al votante, varios autores han intentado demostrar la re-

1 Stokes menciona "Hacia el año 2008, once de los dieciocho países latinoamericanos eran gobernados por presidentes de centro-izquierda o izquierda" (Stokes, 2009, citado en Stoessel, 2014). 
lación existente entre el voto a favor del candidato de gobierno y variables de contexto económico, social y propios del individuo. De esta manera, se ha demostrado que en América Latina los votantes con menores ingresos, así como con menores niveles de educación, suelen mantener su apoyo por el candidato de gobierno (Hunter y Power, 2007; Castillo y Granda, 2014). Considerando lo anterior, partimos de que el votante al buscar su bienestar responderá a los incentivos provenientes del gasto social efectuado por el gobierno, dado que se asume que este tipo de gasto es implementado en su mayoría en contextos de inseguridad económica, provocando que se consolide una base electoral en este sector social.

Para el caso específico de Ecuador, durante el gobierno de la Revolución Ciudadana se evidenció un cambio considerable en las preferencias del votante a lo largo del mandato. Para entender de manera más amplia los factores que influyeron en el voto, y la asignación de recursos del gobierno central a los GADs se plantea un modelo econométrico de datos de panel, en el que se utiliza el gasto social que el gobierno destinó a los cantones durante dos períodos de gobierno, 2010 al 2012 y 2013 al 2017, así como el voto a favor del candidato de gobierno en las elecciones presidenciales de los años 2013 y 2017.

Luego del análisis realizado, se concluye en que el gasto social fue una variable importante que aportó a la estabilidad del gobierno liderado por el expresidente Rafael Correa. Además, se evidencia que este tipo de gasto fue focalizado considerando las variables socio económicas de los cantones logrando que exista un apoyo mayor al candidato del gobierno en los sectores más vulnerables.

\section{Desarrollo}

\section{¿Puede el gobierno actuar de manera táctica?}

Musgrave (1959), define tres funciones principales del Estado, estabilización, asignación y distribución. En el contexto de la teoría de la elección pública, el gobierno puede utilizar los recursos asignados para la función de 
distribución de manera táctica. De ser el caso, la distribución de recursos podría no solo tomar en cuenta el cierre de brechas territoriales, sino que también respondería al deseo de permanencia y del gobierno en curso. De esta manera, los intereses individuales de un gobierno en busca de poder y gloria ${ }^{2}$ pueden alejar la obtención del bienestar común.

Varios autores, han analizado cómo el gobierno puede utilizar sus recursos de manera táctica. Jerosinska (2006) encontró, en Rusia, evidencia que sugería que el gobierno de Vladimir Putin otorgó mayores trasferencias a los grupos que le habían apoyado previamente en las elecciones. Además, demostró que las transferencias se otorgaron a regiones donde había políticos considerados poderosos.

Para entender el comportamiento del gobierno y analizarlo para el caso de nuestro país en este trabajo partiremos de dos hipótesis, la "hipótesis electoral" y la "hipótesis de poder o concesión".

Con respecto a la primera hipótesis, Dixit y Londregan (1996:1113) mencionan dos tipos de redistribución política, la primera que se relaciona con la promoción de la equidad y se rige bajo normativa y la segunda que hace alusión a la redistribución táctica o como se la conoce en la literatura anglosajona "pork barrel".

Si el gobierno hace uso del segundo tipo de redistribución, se podría considerar la existencia de un mercado de votos, en el cual el gobierno, en su esfuerzo por mantenerse en el poder y basándose en los resultados alcanzados en las elecciones previas, transfiere recursos a determinado tipo de votante con el objeto de recibir apoyo político en el futuro incurriendo en costos de monitoreo. ${ }^{3}$

2 "En las democracias actuales, gloria y gobierno son indiscernibles (...) la gloria permite conferir a algo un carácter propiamente político en su doble aspecto divino y humano. El pueblo es esencialmente gloria, aclamación" (Agamben, 2008, citado en Galindo, 2010 ).

3 Zucco (2011) comprueba que no es necesario que el gobierno aplique las estrategias que el clientelismo supone, porque además de ser costosas, las políticas redistributivas llegan al mismo votante y pueden tener un mejor resultado. 
La decisión que tome el gobierno en la forma en que distribuye recursos podrá categorizarlo como un actor riesgo adverso o riesgo amante (Bordalejo, 2005:17).

Lindbeck y Weibull (1987) realizan un modelo en el que existen dos partidos y los votantes votan considerando sus preferencias predefinidas por un partido político y el nivel de consumo que pueden lograr. Los partidos asignan distribuciones de probabilidad a las preferencias de los votantes y ambos partidos asignan la misma probabilidad ya que tienen acceso a la misma información. Además, el presupuesto constituye una restricción para el gasto público por lo que hay sectores no beneficiados. Como resultado obtienen que, siempre que las preferencias sean observables, los candidatos hallarán más rentable invertir sus recursos en los grupos más indecisos, de esta forma, el gobierno será considerado neutral al riesgo.

Por el contrario, si el Gobierno asigna mayor cantidad de recursos a los votantes considerados leales, se asumirá que el gobierno es un agente adverso al riesgo como predicen los autores Cox y McCubbins. Estos autores desarrollan un modelo en el cual se establece que $\mathrm{P}_{\mathrm{ag}}\left(\mathrm{x}_{\mathrm{ag}}, \mathrm{x}_{\mathrm{bg}}\right)$ es la proporción del grupo $\mathrm{G}$ (total de votantes) que apoya a $\mathrm{A}$ (partido político) dado que esta promete una cantidad $\mathrm{x}_{\mathrm{ag}} \mathrm{y}_{\mathrm{bg}}$ (beneficios). Dado que se considera que $\mathrm{P}_{\mathrm{ag}}$ es la proporción del grupo $\mathrm{G}$ que va a apoyar a $\mathrm{A}, \mathrm{P}_{\mathrm{ag}}$ aumenta con $\mathrm{x}_{\mathrm{ag}}$ $\mathrm{y}$ disminuye si $\mathrm{x}_{\mathrm{bg}}$ aumenta. $\mathrm{P}_{\mathrm{ag}}$ es dos veces diferenciable $\mathrm{y}$ cóncava en, $\mathrm{x}_{\mathrm{ag}}$ lo mismo pasa con $\mathrm{B}$, esto significa que ambos partidos tienen rendimientos decrecientes de la inversión en determinados grupos. La existencia de equilibrio en este modelo se garantiza por el supuesto de concavidas. Los autores concluyen que el gobierno usará una estrategia estabilizadora y dirigirá los beneficios a los votantes fieles reforzando la estructura de coalición existente (Cox y MacCubbins, 1986: 373-377).

Por otro lado, con respecto a la hipótesis de "poder”, Grossman (1994) desarrolló un modelo en el que se asume que las transferencias o subvenciones por parte del gobierno compran votos y capital político. Esto es visto como una inversión que asegura el apoyo futuro de estos sectores. Según este 
autor, se esperaría que aquellos políticos más influyentes sean los que reciban mayor cantidad de recursos, por lo que se asume que el gobierno evaluaría la efectividad de los políticos para influenciar en las decisiones de voto (Grossman, 1994).

Para que se considere un sector como políticamente rentable, deberá estar conformado por políticos con trayectoría y niveles altos de aceptación, de manera que invertir en ellos mantenga la coalición con el gobierno central. En el caso ecuatoriano, para medir la aceptación del candidato se utilizó una variable proxy,en la cual se considera si el alcalde del cantón ha sido reelecto.

Además, se considera también como políticamente rentable apoyar a aquellos representantes del partido de gobierno a nivel territorial. De esta manera usaremos como variable de análisis el partido político del alcalde en el poder, basados en los trabajos de Jerosinka (2014), Nazareno, Freille \& Cingolani (2014), entre otros.

\section{¿Y las necesidades territoriales?}

Dentro de la función de distribución, el gobierno utiliza recursos con el objeto de disminuir brechas territoriales. Como parte de estos recursos, está el gasto social, el cual se define como "la cantidad de recursos destinados a financiar políticas relacionadas con salud, educación, vivienda y servicios comunitarios, protección social, actividades recreativas, cultura, religión y protección del ambiente" (CEPAL, sf).

En América Latina se ha demostrado que este gasto social es progresivo, por lo que se asume que debe ir dirigido a los sectores más vulnerables. Para el caso ecuatoriano, Legarda (2014) demostró que para el período 2010 al 2012, el gasto social por motivo de salud, educación y bienestar, se relaciona de manera positiva con las características de contexto del cantón. Es decir, el régimen habría tomado en consideración las necesidades de cada cantón a la hora de asignar el gasto social per cápita.

Sin embargo, ¿puede el gasto social influir en el apoyo al candidato 
de gobierno? La misma autora concluye que "el régimen habría tomado en consideración tanto las necesidades de cada cantón como los resultados electorales a la hora de asignar el gasto social per cápita" (Legarda, 2014:120).

Para este trabajo, al igual que en Legarda se utiliza como variable de análisis principal, el gasto social, ya que "se implementa en contextos que conducen al clientelismo y tiene de beneficiario a la misma gente que es naturalmente objetivo de las políticas redistributivas implementadas en cambios políticos" (Zucco, 2011:11).

Por otro lado, para el caso ecuatoriano no sería adecuado usar otro tipo de transferencias en este análisis considerando el modelo de equitad territorial que consta dentro del marco del Código Orgánico de Planificación y Finanzas Públicas, implementada por el gobierno ecuatoriano a partir del 2010. Autores como Hunter y Power (2007), Zucco (2011), y Legarda (2014) han utilizado esta misma variable en sus estudios previos.

Considerando lo anterior, sugerimos dos posibles comportamientos del gasto social. Primero, el gasto social se distribuye de manera táctica, es decir, no existe un coeficiente significativo en los indicadores de contexto del cantón, como pobreza y educación o, por otro lado, el gasto estuvo bien focalizado y atendió a las necesidades de los cantones. Ambos resultados no son excluyentes.

\section{¿Qué tipo de votante mantiene el apoyo por el candidato de go- bierno?}

Castillo y Granda (2014) definen dos fuentes de motivación para los votantes. Primero están las propias del individuo, es decir sus preferencias, gustos, ideología, percepción política, etnia, género, identificación con el candidato, entre otras, y segundo están los factores de contexto, es decir, las carácterísticas socieconómicas del conjunto poblacional al que pertenece, como por ejemplo el nivel de ingreso y educación. Entre los principales resultados de su investigación los autores encuentran que "mientras mayor es el nivel educativo ... menos probable es el apoyo favorable al partido de 
gobierno" (Castillo y Granda, 2014:59).

Por otro lado, en Brasil, los autores Hunter y Power (2007), muestran que una vez que Luiz Ignácio Lula da Silva alcanza el poder, su base electoral cambia en las siguientes elecciones y consigue el apoyo popular de los sectores más vulnerables económicamente y con menor nivel de escolaridad. De esta manera, demuestra que existe una relación inversa entre el Índice de Desarrollo Humano y el voto a favor del candidato de Gobierno.

Los votantes provenientes de contextos vulnerables tienen horizontes de tiempo cortos, por lo que ellos prefieren beneficios tangibles por sobre las promesas de campaña de entregar más en el futuro. Esto implica que voten por el candidato de gobierno, del que han recibido distintos recursos mientras está en el poder (Kitschelt, 2000, citado en Zucco, 2011).

En el caso de Ecuador, se puede evidenciar a simples rasgos un comportamiento del votante similar al gobierno de Lula da Silva, es decir, a lo largo del gobierno, la base electoral del candidato titular varía considerablemente, estableciéndose en sectores que en principio no votaron por él. Esto podría explicarse por las políticas sociales implementadas en estos sectores.

\section{Ecuador - Mandato de Rafael Correa (2006-2017).}

En Ecuador, antes del gobierno de Correa, se evidenció la destitución y el golpe de Estado de tres presidentes, lo que evidenciaba la inestabilidad política de la época. En relación a lo económico, con respecto al 2000, en el 2006 el PIB había aumentado 3.3\% y la pobreza pasó de 63\% al 45\%, sin embargo, el desempleo y las malas condiciones de los trabajadores no habían mejorado (De la Torre, 2015).

En las elecciones de 2006 "Correa se mostró ante los sectores de izquierda como la encarnación de su lucha en contra del neoliberalismo y de las prácticas corruptas de los políticos”. Su discurso evidenciaba rasgos populistas, entendiendo al populismo como "un discurso político que contrapone al pueblo contra la oligarquía” (De la Torre, 2015:11). De esta manera, el apoyo 
a Correa en las urnas puede ser considerado un acto de democracia plebiscitaria, un acto de fe en la vocación de un jefe (Weber,1969).

En estas elecciones, Correa recibió apoyo principalmente de la Sierra Norte, el Oriente y Galápagos. Sin embargo, a lo largo de su mandato su base electoral cambió drásticamente. Para las elecciones de 2009, 2013 y 2017, el apoyo se concentró principalmente en la región Costa (CNE, 2006, 2009 y 2013).

Esto puede explicarse principalmente por el distanciamiento de grupos ecologista, de izquierda y académicos, el aumento del gasto social y políticas asistencialistas, y el aumento del tamaño del Estado (Valencia, 2009; De la Torre, 2015; Acosta, 2013). Además, los recursos destinados por el gobierno producto del terremoto de 2016, asi como el aumento de políticas de asistencia al sector agrícola, consolidaron la base electoral del candidato de gobierno en la región Costa (Meléndez y Moncagatta $\left.{ }^{4}, 2017\right)$.

A continuación, se presenta el voto a favor del candidato de Gobierno por región:

4 “(...) la atención a la emergencia y el liderazgo político para articular la solidaridad ante eventos de este tipo influyen en el ánimo colectivo y, por lo tanto, en la estabilidad política de los gobiernos de turno" (Meléndez y Moncagatta, 2017: 415). 


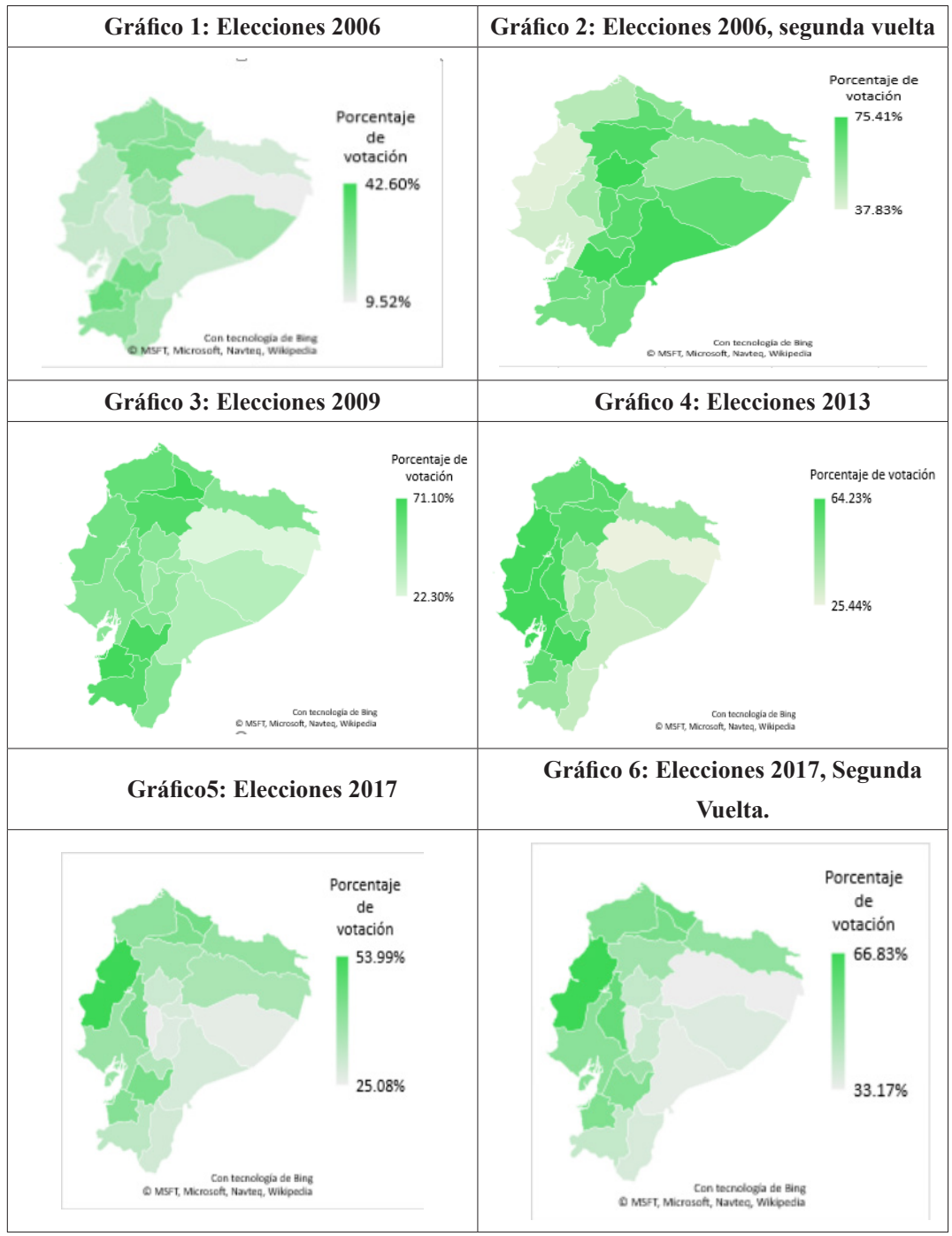

Fuente: CNE, 2006, 2009, 2013 y 2017

\section{Elaborado por: Autora}




\section{El Gasto Social en Ecuador (2006-2017)}

En el gobierno de Rafael Correa, el modelo económico implementado tuvo por objetivo alcanzar el Buen Vivir, lo que "Presupone que las libertades, oportunidades, capacidades y potencialidades reales de los individuos se amplíen de modo que permitan lograr simultáneamente aquello que la sociedad (...) valora como objetivo de vida deseable" (SENPLADES, 2007:54).

Antes de que Correa llegara al poder, el gasto social ecuatoriano era siete veces menor que la región, con 96 USD per cápita frente a 652 USD del promedio regional (CEPAL, 2007). Con el nuevo gobierno, el presupuesto devengado como porcentaje del PIB del sector social pasó de $4.20 \%$ en 2006 a $10.20 \%$ en 2017 . En términos per cápita en 2006 se gastaba por cada persona 142 USD, mientras en el 2017 se llegó a gastar 626 USD (SIICE, 2018).

A manera general, estas políticas, de la mano del aumento de ingresos y boom petrolero lograron mejorar la calidad de vida de la población. De esta manera, un millón cincuenta mil ecuatorianos dejaron de ser pobres por ingresos, entre diciembre de 2006 y diciembre de 2012 (CEPAL, 2012). También el coeficiente de Gini nacional pasó de estar en 0.55 en 2007 a ser 0.46 en el 2017 (INEC, 2017). Por otro lado, la tasa media de matriculación mejoró en todos los niveles, así como el rendimiento de las pruebas de lectura y matemática aumentó alcanzando casi el promedio regional (INEC, 2018).

En el sector salud, la cobertura nacional del seguro de salud pasó de $21.3 \%$ en 2006 a $41.4 \%$ en 2014 . La mortalidad infantil pasó de $20.1 \%$ en 1000 niños en 2012 a $18.4 \%$ en 2015 , y la mortalidad materna pasó de $59.9 \%$ en 2012 a 44.6\% en 2015 (por cada 100.000 nacimientos) (BID, 2018:46).

El aumento del gasto social muestra una ruptura con los anteriores gobiernos, lo que evidencia un cambio de rumbo con respecto al desarrollo social en el país, este cambio afectó positivamente a la población más vulnerable. 


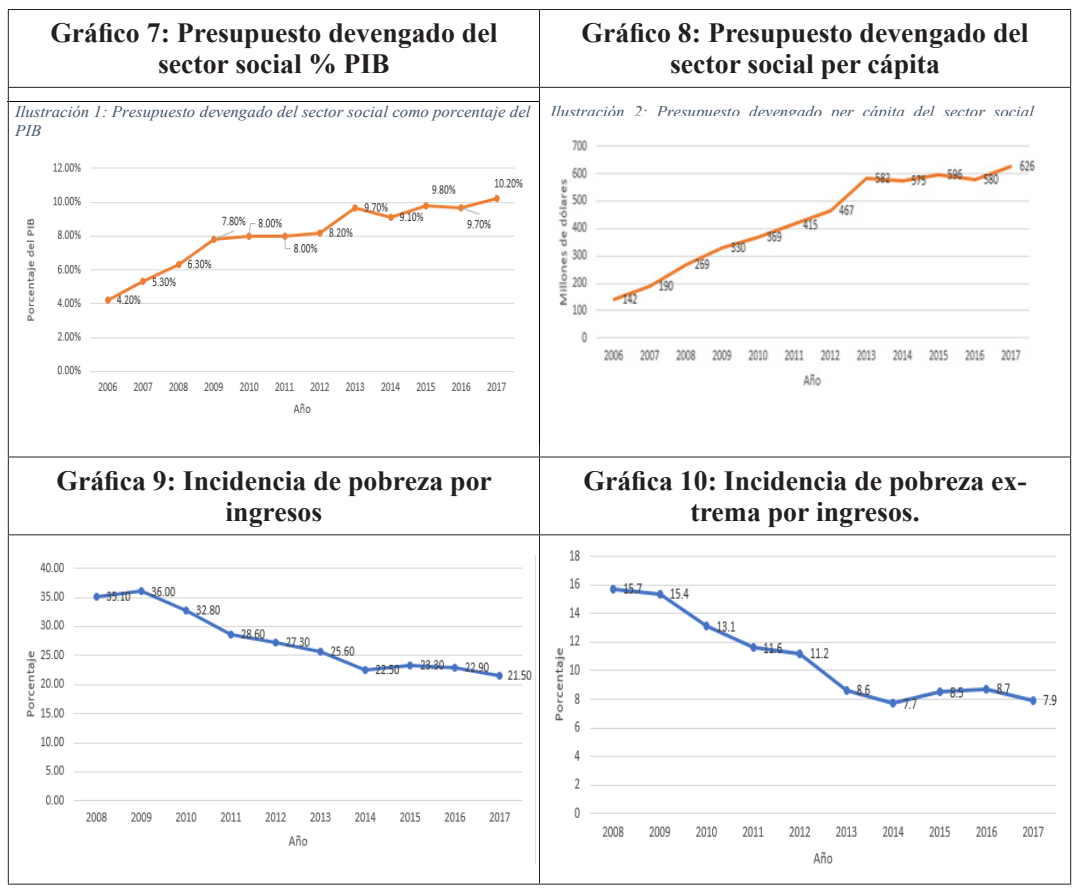

Fuente: Sistema de Indicadores Sociales del Ecuador

Elaboración: Autora 
En síntesis, para entender las variables que influyeron en la estabilidad del gobierno de Rafael Correa, partimos del hecho de considerar que el Gobierno utilizó una redistribución táctica para mantenerse en el poder. Para esto, incurre en costos de monitoreo atados al comportamiento del votante en las elecciones presidenciales previas. Además, también invierte en capital político, al transferir recursos extras a aquellos cantones cuyos alcaldes son considerados "políticamente rentables", es decir, los que hayan sido reelegidos en el cargo, de esta manera, el Gobierno mantendría la coalición que le permite mantenerse en el poder. Por último, se espera también que el gobierno transfiera mayores recursos a los candidatos pertenecientes al partido de gobierno para fortalecer el mismo.

Por otro lado, se toma en cuenta que el marco legal e institucional creado y aplicado durante el gobierno de Rafael Correa planteó las bases para la creación de un Estado garantista de derechos ${ }^{5}$. De manera que, al ver los avances en materia social alcanzados durante su mandato, se espera también que el gasto social haya sido una herramienta que además de mejorar la calidad de vida de las personas, haya influido para fortalecer el apoyo al candidato de gobierno.

Se espera también, que el votante evalúe al candidato de gobierno por las políticas implementadas de manera que aquellos votantes que recibieron más beneficios del gobierno en curso apoyen su reelección, de evidenciar otro comportamiento, este demostraría la insostenibilidad de las coaliciones políticas y la complejidad del ejercicio de gobernar.

\section{Resultados}

Los modelos de datos de panel describen el comportamiento individual a lo largo del tiempo y entre individuos (Katchova, 2013). Los modelos de efectos fijos y aleatorios reconocen que la $\operatorname{Cov}=\left(\mathrm{X}_{\mathrm{it}} ; \mathrm{U}_{\mathrm{it}}\right) \neq 0$, es decir pue-

5 La Constitución del 2008 reconoce al Estado como garantista de derechos. Se establecen además un régimen de Desarrollo y de Buen Vivir. Se crea el Sistema Nacional Descentralizado de Planificación, normado por el Código Orgánico de Organización Territorial (COOTAD) y el Código Orgánico de Planificación y Finanzas Públicas (COPLAFIP). 
den existir factores propios de cada individuo que no se toman en cuenta en el análisis de regresión y dicho componente está reflejado en $\alpha$.

En este trabajo, se realiza un modelo de efectos aleatorios ya que, puede calcular los coeficientes de las variables que no varían en el tiempo, como las relacionadas con la pobreza y escolaridad, además esta decisión se basa en el test de Lagrange de Breuch-Pagan ${ }^{6}$.

La unidad de análisis son los GADs cantonales. De manera general, se trabajó con la información sobre el voto a favor del candidato de gobierno a nivel cantonal en 2013 y 2017 y los planes anuales de inversión territorializados de los años 2010 al 2017. A continuación el detalle:

Tabla 1: Variables de análisis.

\begin{tabular}{|c|c|c|}
\hline \multirow{2}{*}{ Variable } & Variables Dependientes \\
\hline & \multirow{2}{*}{2010 al 2012} & Descripción \\
\hline & & Se usó el Plan \\
& Anual de Inversion, \\
correspondientes \\
Gasto social percápita & a los años 2010, \\
por cantón posterior & 2011 y 2012 (primer \\
a las elecciones & periodo), y 2014, \\
presidenciales (t+1). & \multirow{2}{*}{2014 al 2016} & 2015 2016 (segundo \\
& & periodo), otorgados \\
& & por el MINFIN. \\
& & Se usó montos \\
devengados de gasto \\
& & corriente y capital en \\
& & salud, educación e \\
& & inclusión social. \\
\hline
\end{tabular}

6 La Prob > chi2 para todos los modelos fue menor a 0.05 , rechazando la hipótesis nula que menciona "que no hay diferencia significante entre las unidades", por lo tanto es adecuado elaborar un modelo de efectos aleatorios. 
EL GASTO SOCIAL Y EL VOTO: ANÁLISIS DE LOS PRINCIPALES FACTORES QUE INFLUYEN EN EL COMPORTAMIENTO DEL GOBIERNO Y LOS VOTANTES ECUADOR 2007 -2017

\begin{tabular}{|c|c|c|}
\hline \multirow{2}{*}{$\begin{array}{l}\text { Voto a favor del } \\
\text { candidato de gobierno. }\end{array}$} & $\begin{array}{c}\text { Resultados electorales } 2013 \\
\text { del candidato de gobierno en el } \\
\text { cantón } i\end{array}$ & \multirow{2}{*}{$\begin{array}{c}\text { Bases de datos CNE. } \\
\text { Los datos utilizados } \\
\text { corresponden a } \\
\text { los resultados de } \\
\text { la primera vuelta } \\
\text { electoral. }\end{array}$} \\
\hline & $\begin{array}{c}\text { Resultados electorales } 2017 \\
\text { del candidato de gobierno en el } \\
\text { cantón } i\end{array}$ & \\
\hline \multicolumn{3}{|c|}{ Variables Indenpendientes } \\
\hline Variable & Periodo de analisis & Descripción \\
\hline \multirow{2}{*}{$\begin{array}{l}\text { Partido político del } \\
\text { alcalde del cantón }\end{array}$} & $\begin{array}{l}\text { Elecciones seccionales, } 2004 \text {, } \\
2009\end{array}$ & \multirow{2}{*}{$\begin{array}{c}\text { Dummy, partido de } \\
\text { gobierno }=1\end{array}$} \\
\hline & $\begin{array}{c}\text { Elecciones seccionales } 2009 \text {, } \\
2013\end{array}$ & \\
\hline \multirow{2}{*}{ Alcalde reelecto } & $\begin{array}{l}\text { Elecciones seccionales, } 2004, \\
2009\end{array}$ & \multirow{2}{*}{$\begin{array}{l}\text { Dummy, alcalde } \\
\text { reelegido=1 }\end{array}$} \\
\hline & $\begin{array}{c}\text { Elecciones seccionales, } 2009 \text {, } \\
2013\end{array}$ & \\
\hline $\begin{array}{l}\text { Indicadores } \\
\text { socioeconomicos del } \\
\text { cantón. }\end{array}$ & CENSO 2010: & $\begin{array}{l}\text { Se utilizó; Incidencia } \\
\text { de la pobreza por } \\
\text { NBI, presencia de } \\
\text { analfabetismo, tasa de } \\
\text { asistencia neta básica, } \\
\text { tasa de asistencia } \\
\text { neta de bachillerato, } \\
\text { bachillerato } \\
\text { completo, educación } \\
\text { básica completa en } \\
\text { personas de } 16 \text { años, } \\
\text { secundaria completa } \\
\text { en personas mayores } \\
\text { de } 19 \text { años. }\end{array}$ \\
\hline
\end{tabular}

Fuente y Elaboración: Autora 


\section{Comportamiento del Gobierno}

Para entender el comportamiento del gobierno se consideró la siguiente ecuación:

Gasto $_{10 \text { al I2 }}=\alpha+\beta_{1}\left(\text { Voto }_{\text {Correa }}\right)_{t-1}+\beta_{2}\left(\right.$ Indicadores Socio Económicos $\left._{i}\right)+\beta_{3}$ (partido político del alcalde $\left.{ }_{\mathrm{i}}\right)+\beta 4$ (alcalde reelegido $\left._{i}\right)+\varepsilon_{i t}$

En la cual la unidad de análisis "i” es el cantón, la variable dependiente es el gasto en educación, salud y bienestar social percápita del período de análisis y las variables independiente son el voto a favor de Correa, indicadores socioeconómicos, el partido político y si el alcalde ha sido reelegido.

Considerando lo anterior se obtuvieron los siguientes resultados:

Tabla 2: Variables que influyen en Gasto social 2010-2012

\begin{tabular}{|l|l|l|l|}
\hline & $\begin{array}{c}\text { Gasto pc } \\
\text { salud }\end{array}$ & $\begin{array}{c}\text { Gasto pc } \\
\text { educación }\end{array}$ & $\begin{array}{l}\text { Gasto pc } \\
\text { Bienestar }\end{array}$ \\
\hline \% votos Correa 2009 & 45.96 & $30.9 * *$ & $19.7 * *$ \\
$(42.21)$ & $(13.7)$ & $(26.9)$ \\
\hline Alcalde reelecto & -14.12 & -42.57 & -7.60 \\
& $(11.65)$ & $(38.26)$ & $(7.4)$ \\
\hline Alcalde de PAIS & 5.05 & 39.11 & 4.76 \\
& $(1.09)$ & $(39.54)$ & $(7.67)$ \\
\hline Incidencia de Pobreza & $30.37 * * *$ & $11.06 * * *$ & $18.06 * * *$ \\
& $(7.5)$ & $(2.35)$ & $(45.64)$ \\
\hline $\begin{array}{l}\text { Analfabetismo en personas } \\
\text { de 15 años o más }\end{array}$ & 2.33 & $11.08^{* *}$ & $2.19 * *$ \\
\hline Tasa Neta de Asistencia & $(1.9)$ & $(5.75)$ & $(1.12)$ \\
Primaria & $11.38^{* * * *}$ & 9.24 & 0.03 \\
\hline $\begin{array}{l}\text { Porcentaje de población de } \\
\text { 18 años y más de edad con } \\
\text { bachillerato completo }\end{array}$ & $(4.85)$ & $(10.97)$ & $(0.14)$ \\
\hline
\end{tabular}


EL GASTO SOCIAL Y EL VOTO: ANÁLISIS DE LOS PRINCIPALES FACTORES QUE INFLUYEN EN EL COMPORTAMIENTO DEL GOBIERNO Y LOS VOTANTES ECUADOR 2007 -2017

\begin{tabular}{|l|l|l|l|}
\hline & \multicolumn{1}{|c|}{$\begin{array}{c}\text { Gasto pc } \\
\text { salud }\end{array}$} & $\begin{array}{c}\text { Gasto pc } \\
\text { educación }\end{array}$ & $\begin{array}{c}\text { Gasto pc } \\
\text { Bienestar }\end{array}$ \\
\hline Rho & 0.22 & 0.50 & 0.763 \\
\hline Wald chi2 (1) & 0.042 & 0.001 & 0.023 \\
\hline Número de observaciones & 412 & 412 & 412 \\
\hline
\end{tabular}

Los niveles de significancia son: $* * *<0.01, * *<0.05, *<0.1$

Para el segundo período de análisis se tiene:

Gasto $_{14 \text { al } 17}=\alpha+\beta_{1}\left(\text { Voto Correa }_{i}\right)_{t-1}+\beta_{2}\left(\right.$ Indicadores Socio Económicos $\left._{i}\right)+\beta_{3}$ (partido político del alcalde $\left.{ }_{\mathrm{i}}\right)+\beta 4$ (alcalde reelegido $)+\varepsilon_{i t}$

Tabla 3: Variable dependiente: Gasto social per cápita 2014-2017

\begin{tabular}{|c|c|c|c|}
\hline & $\begin{array}{c}\text { Gasto en } \\
\text { salud }\end{array}$ & $\begin{array}{c}\text { Gasto en } \\
\text { educacion }\end{array}$ & $\begin{array}{c}\text { Gasto en } \\
\text { Bienestar }\end{array}$ \\
\hline \% votos Correa 2013 & 3.58 & 43.97 & 4.68 \\
$(1.32)$ & $(17.72)$ & $(6.83)$ \\
\hline Alcalde reelecto & 0.76 & 49.78 & -43.22 \\
$(2.38)$ & $(6.2)$ & $(26.68)$ \\
\hline Alcalde de PAIS & -1.13 & 12.87 & 11.38 \\
$(2.20)$ & $(4.28)$ & $(3.65)$ \\
\hline Incidencia de Pobreza & $45.43^{* * *}$ & $12.43^{* * *}$ & $36.81^{* * *}$ \\
\hline Analfabetismo en & $(11.68)$ & $(3.2)$ & $(10.08)$ \\
\hline personas de 15 años o & $0.52^{*}$ & 10.64 & $8.88^{* *}$ \\
más & $(6.57)$ & $(7.95)$ & $(4.21)$ \\
\hline Tasa Neta de Asistencia & $1.57^{* * *}$ & 14.95 & 1.33 \\
Primaria & $(0.59)$ & $(14.02)$ & $(6.73)$ \\
\hline Déficit cuantitativo & $0.27 * *$ & $6.02 *$ & $4.69^{* * *}$ \\
$(0.13)$ & $(3.54)$ & $(1.58)$ \\
\hline Rho & 0.78 & 0.82 & 0.27 \\
\hline Wald chi2(1) & 0.00 & 0.046 & 0.07 \\
\hline
\end{tabular}




\begin{tabular}{|l|l|l|l|}
\hline Número de observaciones & 566 & 566 & 566 \\
\hline
\end{tabular}

Los niveles de significancia son: $* * *<0.01, * *<0.05, *<0.1$

Fuente: MINFIN, CNE, INEC, SIN

Elaboración: Autora

Los resultados del modelo econométrico evidencian que en el primer período, el apoyo al candidato de gobierno en las elecciones presidenciales influye de manera positiva en el gasto social ejecutado por el gobierno por motivo de salud y educación, de manera que se cumple con la hipótesis de Cox y McCubbins (1986). Sin embargo, para el segundo período de análisis, esta variable no fue significativa, lo que implica que no se compruebe la hipótesis.

Se puede inferir entonces, que la estrategia del gobierno pudo cambiar, dado que en las siguientes elecciones el gobierno ya estaba suficientemente consolidado para usar los recursos de manera táctica, tal y como Jerociska (2006) demostró en Rusia con el gobierno de Putin .

Por otro lado, es importante considerar la desaceleración de la economía a partir de 2013, lo que implicó que exista un limitante de presupuesto, por lo que, controlar el gasto de manera epecífica se volvió menos plausible, considerando la restricción presupuestaria de las finanzas públicas del gobierno.

Con respecto a la hipótesis de "poder" según la cual el gobierno aportará más recursos considerando la influencia de sus alcaldes en el proceso político (Jarocińska, 2006 y Grossman, 1994), como se evidencia en las estimaciones, esta hipótesis se rechaza para los dos períodos de análisis.

De esta manera, se muestra que no es necesario utilizar estrategias clientelistas porque además de ser costosas, las políticas redistributivas llegan al mismo votante a quien se pretende llegar mediante clientelismo, sin embargo, con las políticas de redistribución, se obtiene un mejor resultado (Zucco, 2011). De esta forma, tanto los votantes como los políticos podrían 
hacerlo mejor en ausencia del clientelismo (Stokes, 2007).

Lo expuesto demuestra que el gobierno no estaba utilizando una estrategia de carácter clientelar y de hecho se enfocó en políticas de redistribución, como el gasto social, lo que le posicionó en el poder durante más de diez años de gobierno.

Los resultados muestran que el gasto social ejecutado por el gobierno durante ambos períodos de análisis se relaciona de manera positiva con las variables de contexto, en especial con la pobreza. Esto permite demostrar que de manera general el gasto social estuvo focalizado en los sectores más vulnerables, confirmando los resultados de Legarda (2016). Se evidencia que la ejecución de recursos sociales fue congruente con el modelo económico y social planteado.

\section{Comportamiento del votante}

Para analizar los factores socioeconómicos y políticos que influyen en el voto a favor del candidato de gobierno se realizó la siguiente ecuación:

$$
\begin{aligned}
\text { Elec }_{13 / 17}= & \alpha+\beta_{1}\left(\text { Gasto Social }_{\mathrm{pci}}\right)_{\mathrm{t}-1}+\beta_{2}\left(\text { Indicadores Socio Económicos }_{\mathrm{i}}\right) \\
& +\beta_{3}\left(\text { Voto Correa }_{\mathrm{i} 09}\right)+\varepsilon_{\mathrm{it}}
\end{aligned}
$$

La variable dependiente es el voto a favor del candidato del gobierno en las elecciones de 2013 y 2017, y las variables independientes son el apoyo recibido en elecciones anteriores, el gasto social recibido por el gobierno y los indicadores de contexto.

Tabla 4: Variable dependiente: Voto a favor de candidato de gobierno elecciones 2013

\begin{tabular}{|c|c|}
\hline VARIABLES INDEPENDIENTES & VOTO CORREA 2013 \\
\hline$\%$ votos Correa 2009 & $0.68^{* * *}$ \\
& $(0.10)$ \\
\hline
\end{tabular}




\begin{tabular}{|c|c|}
\hline Gasto en educacion & -0.0005 \\
& $(0.0004)$ \\
\hline \multirow{2}{*}{ Gasto en salud } & -0.001 \\
& $(0.0007)$ \\
\hline \multirow{2}{*}{ Gasto en bienestar } & 0.00086 \\
& $(0.0001)$ \\
\hline \multirow{2}{*}{ Indice de pobreza } & $0.47^{* * *}$ \\
& $(0.17)$ \\
\hline \multirow{2}{*}{ Déficit cuantitativo de vivienda } & $-0.007 * * *$ \\
& $(0.002)$ \\
\hline Rho & 0.62 \\
\hline Wald chi2 (1) & 0.03 \\
\hline Número de observaciones & $\mathbf{5 6 6}$ \\
\hline
\end{tabular}

Fuente: MINFIN, CNE, INEC, SIN

Elaboración: Autora

Tabla 5: Variable dependiente: Voto a favor de candidato de gobierno elecciones 2017

\begin{tabular}{|c|c|}
\hline VARIABLES INDEPENDIENTES & VOTO LENIN 2017 \\
\hline \multirow{2}{*}{$\%$ votos Correa 2013} & $0.69^{* * *}$ \\
& $(0.02)$ \\
\hline \multirow{2}{*}{ Gasto en educacion } & 0.0001 \\
& $(0.0001)$ \\
\hline \multirow{2}{*}{ Gasto en salud } & -0.0001 \\
& $(0.003)$ \\
\hline \multirow{2}{*}{ Gasto en bienestar } & $0.0017^{* * *}$ \\
& $(0.0005)$ \\
\hline \multirow{2}{*}{ Indice de pobreza } & $0.26^{* * *}$ \\
& $(0.0006)$ \\
\hline \multirow{2}{*}{ Escolaridad } & $-0.007^{* *}$ \\
& $(0.001)$ \\
\hline
\end{tabular}


EL GASTO SOCIAL Y EL VOTO: ANÁLISIS DE LOS PRINCIPALES FACTORES QUE INFLUYEN EN EL COMPORTAMIENTO DEL GOBIERNO Y LOS VOTANTES ECUADOR 2007 -2017

\begin{tabular}{|c|c|}
\hline VARIABLES INDEPENDIENTES & VOTO LENIN 2017 \\
\hline Déficit cualitativo de vivienda & $\begin{array}{c}0.00018^{* * *} \\
(0.0001)\end{array}$ \\
\hline Tasa neta de asistencia a bachillerato & $\begin{array}{c}0.004^{* * *} \\
(0.001)\end{array}$ \\
\hline Rho & 0.84 \\
\hline Wald chi2 (1) & 0.04 \\
\hline Número de observaciones & $\mathbf{5 7 0}$ \\
\hline
\end{tabular}

Fuente: MINFIN, CNE, INEC, SIN

Elaboración: Autora

Los resultados de las estimaciones nos permiten decir, que los electores que votaron por el candidato de gobierno en el 2013 y 2017 confirmaron sus preferencias electorales. Esto implica que existió una especie de lealtad del votante hacia el partido del gobierno como planteó Cox y McCubbins (1986).

Por otro lado, el hecho de que únicamente el gasto en bienestar del período 2014-2017, resulte ser significativo para explicar el voto en favor del candidato de gobierno, no permite dar una conclusión absoluta sobre el efecto del gasto per cápita en salud, educación y bienestar en el voto a favor del candidato de gobierno. Lo que se evidencia, es que a nivel agregado, el votante no parece reaccionar de manera significativa al gasto social per cápita, por lo que el juego entre el gobierno y el votante no sería estable.

Con respecto a las condiciones socioeconómicas, para ambos períodos se demuestra que los cantones con mayor analfabetismo se relacionan de manera positiva con el apoyo a favor del candidato de gobierno. Además se evidencia una relación negativa entre la tasa de asistencia neta de primaria y el voto por el candidato de gobierno para el análisis del primer período. Con respecto a la inseguridad económica, en ambos períodos el voto por el candidato de gobierno se relaciona de manera positiva y significativa con un mayor índice de pobreza en el cantón. 
De esta manera, los cantones con menor educación y mayores niveles de pobreza son los que mantienen la lealtad al gobierno, como Hunter y Power (2007) comprobaron. Por esta razón, la desaceleración económica y corrupción del último período de gobierno de Correa no debilitó el favoritismo del candidato de gobierno en los sectores más vulnerables.

\section{Conclusiones}

Pretender entender el accionar del gobierno y el votante es un reto complejo. Por esa razón, en este trabajo se pretendió (como la teoría económica suele hacer) realizar una abstracción, considerando el papel del gasto social en el juego político.

Considerando los resultados del modelo econométrico, se evidencia que el gasto en salud, educación y bienestar estuvo bien focalizado en los sectores vulnerables, reafirmando lo encontrado en trabajos anteriores (Legarda, 2016). Coincidentemente se ha demostrado que son los sectores más vulnerables los que mantiene su voto por el candidato de gobierno, lo que a manera general nos permite inferir que, este tipo de gasto funciona como un incentivo político eficaz, que además de promover el bienestar social asegura mantener el equilibrio en el juego político, de manera que se alcance estabilidad.

Por otro lado, no se pudo encontrar evidencia concluyente de un comportamiento clientelar del gobierno, lo que puede significar que éste logró consolidarse de manera que no fue necesario usar estas estrategias (Stokes, 2007). En general, se puede decir que la estabilidad del gobierno liderado por el expresidente Rafael Correa, se debió a varios factores, como el carisma del candidato, la situación económica del país, y el modelo económico planteado. Sin embargo, resulta complejo decir que el gobierno tuvo una estrategia clara en la asignación de recursos por motivo de gasto social. 
EL GASTO SOCIAL Y EL VOTO: ANÁLISIS DE LOS PRINCIPALES FACTORES QUE INFLUYEN EN EL COMPORTAMIENTO DEL GOBIERNO Y LOS VOTANTES ECUADOR 2007 -2017

\section{Bibliografía}

Alfonso, G.H. (2010 ). "La gloria y el concepto de lo político en Giorgio Agamben”. Revista de Estudios Sociales [En línea]. Recuperado el 10 de julio del 2020, de http://journals.openedition.org/revestudsoc/14119.

Acosta, A., Cuvi, J., Isch, E., Machado, D., Martínez, E., Muñoz, F. (2013). El país que queríamos. Montecristi Vive. Recuperado el 20 de agosto de 2018, de http://repositorio.uasb.edu.ec/bitstream/10644/4719/1/Soliz, \%20 F-CON018-El\%20pais.pdf

Banco Interamericano de Desarrollo (BID) (2018). Evaluación del Programa de País: Ecuador 2012-2017. Inter-American Development Bank. Recuperado el 10 de agosto de 2018, de https://publications.iadb.org/bitstream/handle/11319/8826/Evaluacion-del-Programa-de-Pais-Ecuador-20122017-FINAL.pdf? sequence $=3$ \&isAllowed $=y$

Buchanan, J. M. (2005). "Elección pública: génesis y desarrollo de un programa de investigación”. RAE: Revista Asturiana de Economía, (33), 203-221.

Castillo, J. G., \& Granda, F. (2014). "Resultados electorales y el contexto socioeconómico: un análisis de las elecciones seccionales en el Ecuador 2009-2014". Revista Democracias, 21-71.

Comisión Económica para América Latina y el Caribe (CEPAL) (2007). Panorama social de América Latina. Santiago de Chile: CEPAL.

Consejo Nacional Electoral. Bases de datos elecciones (2006, 2009, 2013, 2017). Recuperado de http://cne.gob.ec/es/estadisticas/bases-de-datos/ category/320-resultados.

Cox, G. W., \& McCubbins, M. D. (1986). "Electoral politics as a redistributive game". The Journal of Politics, 48(2), 370-389.

De la Torre, C. (2015). De Velasco a Correa: insurrecciones, populismos y elecciones en Ecuador, 1944-2013. Universidad Andina Simón Bolivar 
Ecuador.

Dixit, A., \& Londregan, J. (1996). "The determinants of success of special interests in redistributive politics". the Journal of Politics, 58(4), 1132-1155.

Dieterich, H. S. (2003). El socialismo del siglo XXI. Recuperado el 10 de octubre del 2018 de, https://www.rebelion.org/docs/121968.pdf

Grossman, P. J. (1994). “A political theory of intergovernmental grants". Public Choice, 78 (3-4), 295-303.

Huber, E., \& Dion, M. (2002). "Revolution or contribution? Rational choice approaches in the study of Latin American politics". Latin American Politics and Society, 44 (3), 1-28.

Hunter, W., \& Power, T. J. (2007). "Rewarding Lula: executive power, social policy, and the Brazilian elections of 2006". Latin American Politics and Society, 49 (1), 1-30.

Jarocinska, E. (2006). Political economy of intergovernmental grants. Universitat Pompeu Fabra. Recuperado el 10 de abril de 2018, de http://www. tdx.cat/bitstream/handle/10803/7343/tej.pdf?sequence=1\&isAllowed=y

Katchova (2013). Panel Data Models. Econometrics Academy. Recuperado el 3 de febrero de 2019, de https://sites.google.com/site/econometricsacademy/econometrics-models/panel-data-models

Instituto Nacional de Estadísticas y Censos (16 de enero de 2018). Tabulados de educación. (Base de datos). Recuperado el 12 de septiembre de 2019, de http://www.ecuadorencifras.gob.ec/educacion/

Instituto Nacional de Estadísticas y Censos. (última modificación 23 de febrero de 2017). Tabulados de vivienda.(Base de datos).Recuperado el 12 de septiembre de 2019, de www.ecuadorencifras.gob.ec/.../web.../122016 Tabulados\%20de\%20Vivienda.xlsx 
Instituto Nacional de Estadísticas y Censos. (2017). Reporte de pobreza y desigualdad. Recuperado el 12 de septiembre de 2018, de http://www. ecuadorencifras.gob.ec/documentos/webinec/POBREZA/2017/Junio/Informe\%20pobreza\%20y\%20desigualdad\%20-\%20jun\%202017\%2014072017. pdf

Legarda, V. (2016). "El ciclo político y la economía política del gasto social en el Ecuador". Analitika: revista de análisis estadístico, (12), 107127.

Lindbeck, A., \& Weibull, J. W. (1987). "Balanced-budget redistribution as the outcome of political competition". Public Choice, 52 (3), 273-297.

Ministerio Coordinador de Desarrollo Social (Sf). Sistema de Indicadores Sociales del Ecuador SIISE. Indicadores Sociales del Ecuador. http:// www.siise.gob.ec/siiseweb/

Musgrave, R. (1959). The Theory of Public Finance: A study in public economy. New York: McGraw-Hill.

Meléndez, C., \& Moncagatta, P. (2017). "Ecuador: Una década de correísmo”. Revista de ciencia política (Santiago), 37 (2), 413-448.

Secretaría Nacional de Planificación y Desarrollo (2007). "Plan Nacional de Desarrollo 2007-2010", Quito, Secretaría Nacional de Planificación y Desarrollo. Recuperado el 20 de octubre de 2018, de http://www.senplades. gov.ec/images/stories/descargas/2snp/1pnd/ DLFE-205.pdf

Stokes, S. C. (2007). "Political clientelism". In The Oxford handbook of political science. Recuperado de https://www.oxfordhandbooks. com/view/10.1093/oxfordhb/9780199604456.001.0001/oxfordhb9780199604456-e-031.

Stoessel, S. (2015). "Giro a la izquierda en la América Latina del siglo XXI". Polis [Online]. Recuperado el 05 de julio de 2020 de http://journals. openedition.org/polis/10453 
Valencia, A. (2009). "CRONOLOGIA-Hechos relevantes de la presidencia Correa en Ecuador". Reuters. Recuperado de https://ta.reuters.com/articulo/latinoamerica-elecciones-ecuador-cronolo-idLTASIE53O07Y20090425

Weber, M. (1969). Economía y sociedad, esbozo de sociología comprensiva, Max Weber. Sección de obras de sociología.

Zucco, C. (2008). "The president's 'new'constituency: Lula and the pragmatic vote in Brazil's 2006 presidential elections". Journal of Latin American Studies, 40 (1), 29-49.

Zucco, C. (2011). "Conditional cash transfers and voting behavior: Redistribution and clientelism in developing democracies". Unpublished manuscript. Princeton Universityhttp://pdba.georgetown.edu/Elecdata/Ecuador/ pres06.html 